\title{
GROUPS WITH NO FREE SUBSEMIGROUPS
}

\author{
P. LONGOBARDI, M. MAJ AND A. H. RHEMTULLA
}

\begin{abstract}
We look at groups which have no (nonabelian) free subsemigroups. It is known that a finitely generated solvable group with no free subsemigroup is nilpotent-by-finite. Conversely nilpotent-by-finite groups have no free subsemigroups. Torsion-free residually finite- $p$ groups with no free subsemigroups can have very complicated structure, but with some extra condition on the subsemigroups of such a group one obtains satisfactory results. These results are applied to ordered groups, right-ordered groups, and locally indicable groups.
\end{abstract}

\section{INTRODUCTION}

Let $G$ be a group, and for any pair $(a, b)$ of elements in $G$, let $S(a, b)$ denote the subsemigroup generated by $a$ and $b$. We investigate properties of groups $G$ which contain no free subsemigroup on two generators. In other words, for every pair $(a, b)$ of elements of $G, S(a, b)$ has a relation of the form

$$
a^{r_{1}} b^{s_{1}} \ldots a^{r_{j}} b^{s_{j}}=b^{m_{1}} a^{n_{1}} \ldots b^{m_{k}} a^{n_{k}}
$$

where $r_{i}, s_{i}, m_{i}, n_{i}$ are all nonnegative and $r_{1}$ and $m_{1}$ are positive integers. We shall call $G$ a group without free subsemigroups if it has no free nonabelian subsemigroups; thus taking "free" to mean "free nonabelian." Clearly $G$ has no free subsemigroups if and only if no two generator subgroups of $G$ have free subsemigroup. For this reason there is no loss of generality in assuming that $G$ is finitely generated. Our first result is the following.

Theorem 1. Let $G$ be a finitely generated solvable group. Then $G$ has no free nonabelian subsemigroups if and only if it is nilpotent by finite.

It is well known that $S(a, b)$ is not a free subsemigroup if $\langle a, b\rangle$ is a nilpotent group. In [13] Shalev showed that if $\langle a, b\rangle$ is nilpotent of class $c$, then it satisfies the law $u_{c}=v_{c}$ where the words $\left\{u_{i}\right\},\left\{v_{i}\right\}$ on letters $a, b$ are defined as follows: $u_{0}=a, v_{0}=b$, and for $i \geq 0, u_{i+1}=u_{i} v_{i}$ and $v_{i+1}=v_{i} u_{i}$. Thus if $G=\langle x, y\rangle$ is a periodic extension of a locally nilpotent group and $a, b$ are elements in $G$, then $\left\langle a^{n}, b^{n}\right\rangle$ is nilpotent for some positive integer $n$ and, hence, satisfies the law $u_{c}=v_{c}$ for some $c$ and $\langle x, y\rangle$ does not have a free subsemigroup. The converse is not likely to be true; but with no example known to substantiate this, we leave it as an open question.

Received by the editors February 5, 1994; originally communicated to the Proceedings of the $A M S$ by Ronald M. Solomon.

1991 Mathematics Subject Classification. Primary 20F19. 
Question 1. Let $G=\langle x, y\rangle$ be a group with no free subsemigroups. Is $G$ a periodic extension of a locally nilpotent group?

Even under additional conditions on a group $G$ with no free subsemigroups, the structure of $G$ can be quite complicated. Let $p$ be a prime, $F$ the free group of rank two, and $F / R$ isomorphic to the Gupta-Sidki $p$-group constructed in [7]. Then $F / R$ is an infinite, residually finite $p$-group. Thus $G=F / R^{\prime}$ is a residually torsion-free solvable group. It is also a residually finite $p$-group. For all pairs $(a, b)$ of elements in $G$, there is a relation of type (1) with $j=k=1$. But $G$ is not nilpotent-by-finite. In [5] Grigorchuk constructed interesting examples of finitely generated torsion-free groups of subexponential growth which are not nilpotent-by-finite. These groups, like the group $G=F / R^{\prime}$ described above, are also abelian-by-periodic.

If $(a, b)$ is a pair of elements in $G$ satisfying a relation of type (1), then we call $j+k$ the width of the relation and the sum $r_{1}+\cdots+r_{j}+n_{1}+\cdots+n_{k}$ the exponent of $a$ or $\exp (a)$ in the relation.

Theorem 2. Suppose $G$ is a group and there is a bound $N$ such that for all pairs $(a, b)$ of elements in $G$ there is a relation of the form (1) whose width is at most $N$. Then $G$ is nilpotent if it is residually torsion-free nilpotent.

Note that the group $G=F / R^{\prime}$ quoted above shows that the condition "residually torsion-free nilpotent" cannot be weakened in Theorem 2 . If one looks at groups $G$ where there is a bound $N$ such that for all ordered pairs $(a, b)$ of elements of $G$ there is a relation of the form (1) where $\exp (a)$ is at most $N$, then one can say more about $G$ as the next result shows.

Theorem 3. Suppose $G$ is a locally indicable group and there is a bound $N$ such that for all ordered pairs $(a, b)$ of elements of $G$ there is a relation of the form (1) where $\exp (a)$ is at most $N$. Then $G$ is locally nilpotent-by finite.

One place where the knowledge that $G$ has no free subsemigroup has immediate application is when $G$ is an orderable $(O)$ group or a right orderable $(R O)$ group. We refer the reader to [2] or [4] for basic results and terminology that we use. Recall that $G$ is orderable if there exists a total order relation $\geq$ on $G$ such that for all $a, b, h, g$ in $G, a \geq b$ implies $h a g \geq h b g$; equivalently, if there exists a normal subset $P$ in $G$ such that $P P=P, P \cup P^{-1}=G$, and $P \cap P^{-1}=\{e\} . \quad G$ is right orderable if there exists a total order relation $\geq$ on $G$ such that for all $a, b, g$ in $G, \quad a \geq b$ implies $a g \geq b g$, equivalently, if there exists a subset $P$ in $G$ such that $P P=P, \quad P \cup P^{-1}=G$, and $P \cap P^{-1}=\{e\}$. We shall show that if $G$ is orderable and has no free subsemigroup on two generators, then all the convex subgroups are normal in $G$ under any order on $G$. And if $G$ is right orderable and has no free subsemigroup on two generators, then under any right order on $G$ the set of convex subgroups form a series with torsion-free abelian factors; and, in particular, $G$ is locally indicable. Our result thus extends the well-known result that nilpotent-by-finite right orderable groups are locally indicable. It also extends a recent result of Kropholler in [10] that the convex subgroups of a right-ordered supramenable group form a series with torsion-free abelian factors. This follows since supramenable groups contain no free semigroups [14, p. 189]. As corollaries of Theorems 2 and 3, we get 
Theorem 4. If $G$ is an O-group and there is a bound $N$ such that for all pairs $(a, b)$ of elements in $G$ there is a relation of the form (1) whose width is at most $N$, then $G$ is nilpotent of class bounded by $N$.

Theorem 5. If $G$ is an RO-group and there is a bound $N$ such that for all ordered pairs $(a, b)$ of elements of $G$ there is a relation of the form (1) where $\exp (a)$ is at most $N$, then $G$ is locally nilpotent-by finite.

Recently Grigorchuk and Machi showed in [6] that the torsion-free groups of subexponential growth constructed by Grigorchuk in [5] that we referred to earlier are also right orderable. Thus a finitely generated $R O$-group of subexponential growth need not be nilpotent-by-finite. We do not know if a finitely generated $O$-group of subexponential growth must be nilpotent.

We thank Dr. Shirvani for pointing out to us that Theorem 1 was proved by Rosenblatt in [12]. His proof depends heavily on the works of Wolf [15] and Milnor [11]; our proof is short and direct. For this reason we have included the proof in this paper. There is a close similarity between our proof of Lemma 4 and that of Lemma 2 by Bass in [1].

\section{Proofs}

Lemma 1. If $G$ has no free subsemigroups, then for all $a, b$ in $G,\left\langle a^{\langle b\rangle}\right\rangle$ is finitely generated.

Proof. Consider the semigroup $S\left(b, b^{a}\right)$ generated by $b$ and $b^{a}$. By hypothesis,

$$
b^{r_{1}}\left(b^{a}\right)^{s_{1}} \ldots b^{r_{j}}\left(b^{a}\right)^{s_{j}}=\left(b^{a}\right)^{m_{1}} b^{n_{1}} \ldots\left(b^{a}\right)^{m_{k}} b^{n_{k}},
$$

where $r_{i}, s_{i}, m_{i}, n_{i}$ are nonnegative integers and $r_{1}$ and $m_{1}$ are positive. Hence

$$
\left(a^{-1}\right)^{b^{\lambda_{1}}} a^{b^{\lambda_{2}}}\left(a^{-1}\right)^{b^{\lambda_{3}}} \ldots a^{b^{\lambda_{u}}} b^{-\lambda_{u}}=\left(a^{-1}\right) a^{b^{\mu_{2}}}\left(a^{-1}\right)^{b^{\mu_{3}}} \ldots a^{b^{\mu_{v-1}}} b^{-\mu_{v}}
$$

where $\lambda_{u}<\cdots<\lambda_{1}<0$ and $\mu_{v}<\cdots<\mu_{2}<0$. Let $\lambda=\lambda_{u}, \mu=\mu_{v}$. If $\lambda \neq \mu$, then $b^{\lambda-\mu} \in\left\langle a^{\langle b\rangle}\right\rangle$, which is then finitely generated; and we are done. So assume $\lambda=\mu$. Then $a \in\left\langle a^{b^{-1}}, \ldots, a^{b^{\lambda}}\right\rangle$. By replacing $b$ with $b^{-1}$ we similarly get $a \in$ $\left\langle a^{b}, \ldots, a^{b^{\nu}}\right\rangle$ for some $\nu>0$. Thus $\left\langle a^{b^{\nu}}, \ldots a^{b}, a, a^{b^{-1}}, \ldots, a^{b^{\lambda}}\right\rangle=\left\langle a^{\langle b\rangle}\right\rangle$.

The next result appears in [9], but we include the proof here since it is very short.

Lemma 2. Let $G$ be a finitely generated group. If $H \triangleleft G, \quad G / H$ is cyclic, and $\left\langle a^{\langle b\rangle}\right\rangle$ is finitely generated for all $a, b$ in $G$, then $H$ is finitely generated.

Proof. For some $g \in G$ we can write $G=H\langle g\rangle$. Since $G$ is finitely generated, there exist $h_{1}, \ldots, h_{r}$ in $H$ such that $G=\left\langle h_{1}, \ldots, h_{r}, g\right\rangle$ and $H=$ $\left\langle h_{1}, \ldots, h_{r}\right\rangle^{G}$. For each $i=1, \ldots, r,\left\langle h_{i}^{\langle g\rangle}\right\rangle$ is finitely generated, say, $\left\langle h_{i}^{\langle g\rangle}\right\rangle=$ $\left\langle h_{i 1}, \ldots, h_{i d(i)}\right\rangle$. Then $H=\left\langle h_{i \ell(i)} ; 1 \leq i \leq r, 1 \leq \ell(i) \leq d(i)\right\rangle$.

Corollary 3. Let $G$ be a finitely generated group with no free subsemigroups. Then for every positive integer $n$, the $n$th derived subgroup $G^{(n)}$ is finitely generated. In particular if $G$ is solvable, then it is polycyclic.

Proof. This follows directly from Lemma 1 and Lemma 2.

Lemma 4. Let $G=A \rtimes T$, the split extension of a finitely generated torsion-free abelian group $A$ by infinite cyclic group $T=\langle t\rangle$. If $T$ acts rationally irreducibly on $A$ and $G$ has no free subsemigroups, then $G$ is abelian-by-finite. 
Proof. Let $V=A \otimes_{\mathbb{Z}} \mathbb{Q}$. Then $V$ is an irreducible $Q T$-module and by Schur's Lemma, $D=\operatorname{End}_{\mathscr{Q} T} V$ is a division ring of finite dimension over $\mathbb{Q}$. Now the image of $T$ in End $V$ lies in $D$ and generates $D$. Hence $D$ is an algebraic number field. As a $D$-space, $V$ is one dimensional. Let $\alpha$ be the image of $t$ in $D$. Then we can identify $V$ with $\mathbb{Q}(\alpha)$ under addition and the action of $t$ on $V$ being that of multiplication by $\alpha$. If $\alpha$ is a root of 1 , then $t^{n}$ acts trivially on $V$ and hence the subgroup $\left\langle A, t^{n}\right\rangle$ is abelian of finite index in $G$. If $a$ is not a root of unity, then $D$ can be embedded in $\mathbb{C}$ so that $|\alpha|<1$ (see $[8$, p. 102]). By taking a power of $\alpha$, if necessary, we may assume that $|\alpha|<\frac{1}{4}$.

Take any $b \neq e$ in $A$ and consider the semigroup $S\left(t, t^{b}\right)$. By hypothesis there exist positive integers $p, q, r_{1}, \ldots, r_{p}, s_{1}, \ldots, s_{p-1}, u_{2}, \ldots, u_{q-1}$, $v_{1}, \ldots, v_{q}$ and $s_{p}, u_{q}$ nonnegative such that

$$
t^{r_{1}}\left(t^{b}\right)^{s_{1}} \ldots t^{r_{p}}\left(t^{b}\right)^{s_{p}}=\left(t^{b}\right)^{v_{1}} t^{u_{2}} \ldots\left(t^{b}\right)^{v_{q}} t^{u_{q}} .
$$

Note that $\sum_{i=1}^{p} r_{i}+\sum_{i=1}^{p} s_{i}=\sum_{i=2}^{q} u_{i}+\sum_{i=1}^{q} v_{i}$ since $G / A$ is infinite. If $\beta$ corresponds to $b$ in the isomorphism of $V$ and $\mathbb{Q}(\alpha)$, then the above equality translates into

$$
\beta \sum_{i=1}^{j} \pm \alpha^{\lambda_{i}}=\beta \sum_{i=1}^{k} \pm \alpha^{\mu_{i}}
$$

where $0<\lambda_{1}<\cdots<\lambda_{j} ; \quad 0=\mu_{1}<\mu_{2}<\cdots<\mu_{k}$, and $j, k$ are some positive integers. Since $|\alpha|<\frac{1}{4}, \quad\left|\sum_{i=1}^{j} \pm \alpha^{\lambda_{i}}\right| \leq \sum_{i=1}^{j}\left(\frac{1}{4}\right)^{\lambda_{i}}<\frac{1}{2}$. On the other side, $\left|\sum_{i=1}^{k} \pm \alpha^{\mu_{i}}\right| \geq 1-\sum_{i=2}^{k} \frac{1}{4}^{\mu_{i}}>\frac{1}{2}$, giving a contradiction. Thus $G$ is abelian-by-finite.

Proof of Theorem 1. Let $G$ be a finitely generated solvable group with no free subsemigroup. We use induction on the solvability length of $G$ to show that $G$ is nilpotent-by-finite. Clearly there is nothing to prove if $G$ is abelian. Hence, using induction, we may assume that $G$ is abelian-by-nilpotent-by-finite. Taking a subgroup of finite index in $G$, if necessary, we may assume that $G$ is abelianby-nilpotent. By Corollary 3 , we know that $G$ is polycyclic. Thus, again passing to a subgroup of finite index, if necessary, we may assume that $G$ has a finitely generated torsion-free normal abelian subgroup $A$ and $G / A$ is torsion-free nilpotent. Now, there is a central series $A=A_{0} \triangleleft A_{1} \triangleleft \ldots \triangleleft A_{s}=G$ from $A$ to $G$ with infinite cyclic factors. Say $A_{i}=A_{i-1}\left\langle t_{i}\right\rangle, i=1, \ldots, s$. It suffices to show that $\left\langle A, t_{i}^{n_{i}}\right\rangle$ is nilpotent for some $n_{i}>0$; for then $\left\langle A, t_{1}^{n_{1}}, \ldots, t_{s}^{n_{s}}\right\rangle$ is nilpotent and of finite index in $G$, as is required to show.

In order to show that $\left\langle A, t_{i}^{n_{i}}\right\rangle$ is nilpotent for some $n_{i}>0$, consider the series $1=A_{i 0} \triangleleft \ldots \triangleleft A_{i m(i)}=A$ where $A_{i j}$ are isolated subgroups of $A$, normalized by $t_{i}$, and $A_{i j+1} / A_{i j}$ is of minimal rank. Apply Lemma 4 to $\left\langle A_{i j+1}, t_{i}\right\rangle / A_{i j}$ to get $\left[A_{i j+1}, t_{i}^{n_{i}}\right] \leq A_{i j}$ for some $n_{i}>0$ and all $j=$ $0, \ldots, m(i)$. Then $\left\langle A, t_{i}^{n_{i}}\right\rangle$ is nilpotent of class at most $m(i)$. This completes the proof.

Proposition 5. Suppose that $G$ is a torsion-free nilpotent group. If there is a bound $N$ such that for all pairs $(a, b)$ of elements in $G$ there is a relation of 
the form (1) whose width is at most $N$, then $G$ is nilpotent whose class is bounded by a function of $N$.

Proof. Let $G$ be nilpotent of class $c$. Then $\gamma_{[c / 2]}(G)$ is abelian, where [c/2] equals $c / 2$ if $c$ is even and $c+1 / 2$ if $c$ is odd. Let $A$ denote the isolator of $\gamma_{[c / 2]}(G)$. Then $A$ is also abelian since $G$ is torsion-free. Now, for any $a \in A$ and $g \in G$, consider $S(g, a)$ to obtain the equality

$$
a^{r_{1}} g^{s_{1}} \ldots a^{r_{j}} g^{s_{j}}=g^{m_{1}} a^{n_{1}} \ldots g^{m_{k}} a^{n_{k}}
$$

where $j+k \leq N$. We treat $A$ as a $\mathbb{Z}\langle g\rangle$-module and show that $A(g-1)^{N}=0$. If $g \in A$, then $A(g-1)=0$ and we are done. So assume $g \notin A$. Now the relation (2) yields

$$
a \sum_{i=1}^{j} r_{i} g^{\mu_{i}}=a \sum_{i=1}^{k} n_{i} g^{\lambda_{i}}
$$

where $0=\mu_{1}<\mu_{2}<\cdots<\mu_{j}$ and $0<\lambda_{1}<\cdots<\lambda_{k}$. Let $\sum_{i=1}^{j} r_{i} x^{\mu_{i}}-\sum_{i=1}^{k} n_{i} x^{\lambda_{i}}=$ $q(x)$, and let $A_{1}=A \otimes_{\mathbb{Z}} \mathbb{Q}$. Treat $g$ as an operator on $A_{1}$ to get $a q(g)=0$. Since $\left\langle A_{1}, g\right\rangle$ is also nilpotent of class at most $c,(g-1)^{c}$ annihilates $a$ as does $q(g)$. Now if $(x-1)^{e}$ divides $q(x)$, then $e \leq N$ for we have $q(1)=$ $0, q^{\prime}(1)=0, \ldots, q^{e-1}(1)=0$ where $q(x)=\sum_{i=1}^{t} c_{i} x^{\nu_{i}}$ where $t \leq N, c_{i} \neq 0$. Then

$$
\left(\begin{array}{ccc}
1 & \cdots & 1 \\
\nu_{1} & \cdots & \nu_{t} \\
\nu_{1}^{2} & \cdots & \nu_{t}^{2} \\
& \vdots & \\
\nu_{1}^{e-1} & \cdots & \nu_{t}^{e-1}
\end{array}\right)\left(\begin{array}{c}
c_{1} \\
\vdots \\
c_{t}
\end{array}\right)=\left(\begin{array}{c}
0 \\
\vdots \\
0
\end{array}\right) \text {. }
$$

If $e \geq N$, then the only solution to the above system is $c_{i}=0$ for all $i=1, \ldots, t$ since $t \leq N$. This, in turn, would imply that $q(x)=0$, a contradiction. Thus $a(g-1)^{N}=0$ for all $a \in A, g \in G$. Reverting to the multiplicative notation of the group $G$, we have

$$
[A, \underbrace{g, \ldots, g}_{N}]=1 \text {. }
$$

Since $G$ is torsion-free, it follows from a result of Zelmanov (see [16, p. 166]) that $A$ lies in $\zeta_{f(N)}(G)$, the $f(N)$ th center of $G$, where $f(N)$ is a function of $N$ and independent of the number of generators of $G$. Thus the nilpotency class of $G$ is at most $[c / 2]+f(N)$ and hence $c \leq 2 f(N)$.

It is worth noting here that the method applied in the proof of Proposition 5 is general enough to be useful in other situations. We mention one such case. Suppose $G$ is a torsion-free locally solvable group and, for some fixed positive integer $N,\langle x, y\rangle=(\langle x\rangle\langle y\rangle)^{N}$ for all $x, y$ in $G$. Then $G$ is nilpotent of class bounded by a function of $N$.

Proof of Theorem 2. Since $G$ is residually torsion-free nilpotent, there exists a descending central series $G=G_{0}>G_{1}>\cdots$ where $\bigcap_{i=1}^{\infty} G_{i}=1$ and $G / G_{i}$ is 
torsion-free nilpotent for all $i$. By Proposition 5, there is some integer $f(N)$ such that $\gamma_{f(N)+1}(G) \subseteq G_{i}$ for all $i$ and hence $\gamma_{f(N)+1}(G)=1$.

Lemma 6. If $A$ is a $\mathbb{Z}$-torsion-free $\mathbb{Z}\langle g\rangle$-module, $q(x) \in \mathbb{Z}[x]$ is a polynomial of degree $N$, and for some $a \in A, \quad a(q(g))=a\left(g^{n}-1\right)=0$ for some $n>0$, then there exists a positive integer $m=m(N)$, independent of $n$, such that $a\left(g^{m}-1\right)=0$.

Proof. Consider $q(x)$ and $x^{n}-1$ as elements of $\mathbb{Q}[x]$. Since $x^{n}-1=$ $\prod_{d \mid n} \theta_{d}(x)$, where $\theta_{d}(x)$ are the $d$ th cyclotomic polynomials of degree $\psi(d)$ which are irreducible in $\mathbb{Q}[x]$, the greatest common divisor $\left(q(x), x^{n}-1\right)=$ $\theta_{d_{1}}(x) \ldots \theta_{d_{r}}(x)$ for some $d_{i} \mid n$. Now there are only finitely many integers $d_{i}$ such that $\psi\left(d_{i}\right) \leq N$. Let $d(x)$ be the product of these $\theta_{d_{i}}(x)$, and let $m$ be the least integer such that $d(x)$ divides $x^{m}-1$. Then for some positive integer $s, a\left(s\left(g^{m}-1\right)\right)=0$, since $A$ is a $\mathbb{Z}\langle g\rangle$-module rather than $\mathbb{Q}\langle g\rangle$-module. But since $A$ is $\mathbb{Z}$-torsion-free, $s\left(a\left(g^{m}-1\right)\right)=0$ implies $a\left(g^{m}-1\right)=0$. This completes the proof.

Proposition 7. Suppose that $G$ is a torsion-free solvable group. If there exists a bound $N$ such that for all ordered pairs $(a, b)$ of elements in $G$ there is $a$ relation of the form (1) in which $\exp (a)$ is at most $N$, then the exponent of $G / \operatorname{Fitt}(G)$ and the nilpotency class of $\operatorname{Fitt}(G)$ are bounded by a function of $N$.

Proof. There is no loss of generality in assuming that $G$ is finitely generated since we shall show that $G / \operatorname{Fitt}(G)$ has bounded exponent and the nilpotency class of $\operatorname{Fitt}(G)$ is bounded. The bound on Fitt $(G)$ is obtained from Proposition 5. By Theorem 1, $G$ is nilpotent-by-finite. Let $A$ be a normal abelian subgroup of $G$. Then for any $a \in A$ and $g \in G$ consider the ordered pair $(g, a)$ to obtain the equality

$$
a^{r_{1}} g^{s_{1}} \ldots a^{r_{j}} g^{s_{j}}=g^{m_{1}} a^{n_{1}} \ldots g^{m_{k}} a^{n_{k}}
$$

where $\sum_{i=1}^{j} s_{i}+\sum_{i=1}^{k} m_{i} \leq N$. We consider $A$ as $\mathbb{Z}\langle g\rangle$-module and show that $a\left(g^{m}-1\right)=0$ for some $m=m(N)$. Equation (3) in additive notation yields

$$
a \sum_{i=1}^{j} r_{i} g^{\mu_{i}}=a \sum_{i=1}^{k} n_{i} g^{\lambda_{i}}
$$

where $0=\mu_{1}<\mu_{2}<\cdots<\mu_{j} ; 0<\lambda_{1}<\cdots<\lambda_{k}$, and $\mu_{j}+\lambda_{k} \leq N$. Let $q(x)=\sum_{i=1}^{j} r_{i} x^{\mu_{i}}-\sum_{i=1}^{k} n_{i} x^{\lambda_{i}}$ so that $a \cdot q(g)=0$. Since $G$ is nilpotentby-finite, $a\left(g^{n}-1\right)^{c}=0$ for some positive integers $n$ and $c$. If $c=1$, then by Lemma $6, a\left(g^{m}-1\right)=0$ where $m$ depends only on $N$. If $c>1$, then replace $a$ by $a\left(g^{n}-1\right)^{c-1}$ to get $a\left(g^{n}-1\right)^{c-1}\left(g^{m}-1\right)=0$ and hence $a\left(g^{m}-1\right)\left(g^{n}-1\right)^{c-1}=0$. Use induction on $c$ to get $a\left(g^{m}-1\right)^{c}=0$.

Let $F=\operatorname{Fitt}(G)$. We show that $G^{m} \subseteq F$. Since $G$ is nilpotent-by-finite by Theorem $1, F$ is finitely generated so that the terms of the upper central series of $F$ are all finitely generated isolated subgroups of $F$. Let $Z_{i}$ denote the $i$ th 
center of $F$. Then $Z_{i}\left(g^{m}-1\right)^{c_{i}} \subseteq Z_{i-1}$ where $c_{i} \in \mathbb{N}$. Thus $F$ lies in some term of the upper central series of $F G^{m}$. But if $F \neq F G^{m}$, then let $B / F$ be a nontrivial normal abelian subgroup of $F G^{m} / F$. Then $B \subseteq \operatorname{Fitt}(G)=F$, a contradiction. Now apply Theorem 2 to $F$ to get a bound on the nilpotency class of $F$.

Proof of Theorem 3. We assume that $G$ is finitely generated and show that it is nilpotent-by-finite. Let $R$ be the torsion-free solvable residual of $G$. Then $R=\bigcap_{i=1}^{\infty} G_{i}$ where $G / G_{i}$ is torsion-free solvable. By Proposition $7, G_{i} G^{m} / G_{i}$ is nilpotent of class $n$ with $n$ and $m$ depending only on $N$. Thus $G / R$ is solvable and by Corollary $3, R$ is finitely generated. If $R \neq 1$, then, by hypothesis, $R / R^{\prime}$ is infinite so that $G / J$ is torsion-free solvable where $J$ is the isolator of $R^{\prime}$ in $R$, and $J \neq R$, which is a contradiction.

Lemma 8. Let $\geq$ be a (two-sided) total order on an O-group $G$. If $G$ has no free subsemigroups, then the convex subgroups under $\geq$ are normal in $G$.

Proof. Let $C$ be a convex subgroup under $\geq$, and suppose that $C^{g} \neq C$ for some $g \in G$. We may assume that $C^{g} \supseteq C$ since convex subgroups are nested. Thus there exists $a$ in $C$ such that $a^{g}$ is not in $C$. But then $a^{g^{n+1}}$ is not in $C^{g^{n}}$ for all $n \geq 0$. But $\left\langle a^{\langle g\rangle}\right\rangle$ is finitely generated by Lemma 1. Thus $a^{g^{n+1}} \in\left\langle a, a^{g}, \ldots, a^{g^{n}}\right\rangle$ for some $n$, and hence $a^{g^{n+1}} \in C^{g^{n}}$, a contradiction.

Proof of Theorem 4. Let $\geq$ be a total order on $G$. Let $C \mapsto D$ be a jump in the set of convex subgroups of $G$ under $\geq$; then $D / C$ is order isomorphic to a subgroup of the additive group of reals and every $g$ in $G$ induces an orderpreserving automorphism of $D / C$ (see $[4$, p. 50]). Since the order-preserving automorphisms of an Archimedean-ordered group form a subgroup of the multiplicative group of positive reals, it follows that the centralizer $C_{G}(D / C)$ of $D / C$ in $G$ contains $G^{\prime}$ and is an isolated subgroup of $G$. Let $J$ be the isolator of $G^{\prime}$ in $G$. Then $[D, J] \subseteq C$.

Since we shall show that $G$ is nilpotent of class bounded by $N$, independent of the number of generators of $G$, it suffices to assume that $G$ is finitely generated. Then by Corollary $3, J$ is also finitely generated. Now order $J$ by taking the restriction of order $\geq$. Then the convex subgroups of $J$ are $C \cap J$, where $C$ is convex in $G$ under $\geq$. This order on $J$ is a $G$-order in that the positive cone is invariant under conjugation by elements of $G$. We use this order on $J$ and extend it to an order on $G$ by making $J \mapsto G$ a convex jump. Since $J$ is finitely generated, there is $J_{1} \leq G$ such that $J_{1} \longmapsto J . \quad\left(J_{1}\right.$ is simply the largest convex subgroup of $G$ that does not contain the finite set that generates $J$.) Similarly $J_{1}$ is finitely generated by Corollary 3 and there is a jump $J_{2} \nrightarrow J_{1}$.

Continue this process, and let $K=\bigcap_{i=1}^{\infty} J_{i}$.

Then $J / K$ is residually torsion-free nilpotent and hence nilpotent by Theorem 2 . Thus $G / K$ is soluble and hence nilpotent-by-finite by Theorem 1. But a nilpotent-by-finite $O$-group is nilpotent.

Hence $G / K$ is nilpotent and the nilpotency class is bounded by a function of $N$ as in Proposition 5. Thus $K=J_{m}$ for some $m$ and $J_{m}=1$. 
Lemma 9. Let $\geq$ be a right order on an RO-group $G$, and let $P=\{g \in G ; g>$ $e\}$. If $P$ has no free subsemigroup, then the set of convex subgroups under $\geq$ form series from $\{e\}$ to $G$ with torsion-free abelian factors. In particular $G$ is locally indicable.

Proof. Let $a, b$ be any elements in $P$. We first show that $a^{n} b>a$ for some positive integer $n$. If $b>a$, then $a^{n} b>b>a$ for all $n \geq 0$, so assume $a>b$. By hypothesis

$$
a^{r_{1}} b^{s_{1}} \ldots a^{r_{j}} b^{s_{j}}=b^{m_{1}} a^{n_{1}} \ldots b^{m_{k}} a^{n_{k}}
$$

for some non-negative integers $r_{i}, s_{i}, m_{i}, n_{i}$ where $s_{j}$ and $n_{k}$ are positive. If $a>a^{n} b$ for all $n \geq 0$, then $a^{r_{1}} b^{s_{1}}=\left(a^{r_{1}} b\right) b^{s_{1}-1}<a b^{s_{1}-1}<\cdots<$ $a b<a$. Continue in this fashion to get $a^{r_{1}} b^{s_{1}} \ldots a^{r_{j}} b^{s_{j}}<a$. On the other side $b^{m_{1}} a^{n_{1}} \ldots b^{m_{k}} \geq e$ so that $b^{m_{1}} a^{n_{1}} \ldots b^{m_{k}} a^{n_{k}} \geq a^{n_{k}} \geq a$, resulting in a contradiction. Now a right order $\geq$ where for each ordered pair $(a, b)$ of elements in $P$ there exists some $n \geq 0$ such that $a^{n} b>a$ is called a $C$-order. It was shown by Conrad in [3] that if $\geq$ is a $C$-order, then the set of convex subgroups of $G$ under $\geq$ forms a system in Malcev terminology (series in P. Hall terminology) with torsion-free abelian factors. Thus every nontrivial finitely generated subgroup of $G$ has an infinite cyclic quotient and $G$ is locally indicable.

Proof of Theorem 5. This follows from Lemma 3 and Theorem 3.

\section{REFERENCES}

1. H. Bass, The degree of polynomial growth of finitely generated nilpotent groups, Proc. London Math. Soc. (3) 25 (1972), 603-614.

2. R. Botto Mura and A.H. Rhemtulla, Orderable groups, Dekker, 1977.

3. P.F. Conrad, Right ordered groups, Michigan Math. J. 6 (1959), 267-275.

4. L. Fuchs, Partially ordered algebraic systems, Pergamon Press, 1963.

5. R. I. Grigorchuk, On the growth degrees of p-groups and torsion-free groups, Math. Sb. 126 (1985), 194-214; English transl. Math. USSR-Sb. 54 (1986), 347-352.

6. R. I. Grigorchuk and A. Machi, An intermediate growth automorphism group of the real line, preprint.

7. N. Gupta and S. Sidki, Some infinite p-groups, Algebra i Logika 22 (1983), 584-589.

8. E. Hecke, Lectures on the theory of algebraic numbers, Translated by George U. Brauer and Jay R. Goldman, Springer-Verlag, 1981.

9. Y. K. Kim and A. H. Rhemtulla, Weak maximality condition and polycyclic groups.

10. P. H. Kropholler, Amenability and right orderable groups, Bull. London Math. Soc. 25 (1993), 347-352.

11. J. Milnor, Growth of finitely generated solvable groups, J. Differential Geom. 2 (1968), 447-449.

12. J. M. Rosenblatt, Invariant measures and growth conditions, Trans. Amer. Math. Soc. 197 (1974), 33-53.

13. A. Shalev, Combinatorial conditions in residually finite groups, II, J. Algebra 157 (1993), $51-62$

14. S. Wagon, The Banach-Tarski paradox, Cambridge University Press, 1985. 
15. J. Wolf, Growth of finitely generated solvable groups and curvature of Riemannian manifolds, J. Differential Geom. 2 (1968), 421-446.

16. E. I. Zelmanov, On some problems of group theory and lie algebras, Math. USSR-Sb. 66 (1990), 159-168.

Dipartimento di Matematica e Applicazioni, R. Caccioppoli, Universita di Napoli, 80126 NAPLES, ITALY

E-mail address: longobar@matna1.dma.unina.it

E-mail address: maj@matna1.dma.unina.it

Department of Mathematics, University of Alberta, Edmonton, Alberta, Canada T6G 2G1

E-mail address: akbar@malindi.math.ualberta.ca 